\title{
130.
}

\section{DEUXIÈME MÉMOIRE SUR LES FONCTIONS DOUBLEMENT PÉRIODIQUES.}

[From the Journal de Mathématiques Pures et Appliquées (Liouville), tom. xIx. (1854), pp. 193-208: Sequel to Memoir t. x. (1845), 25.]

JE vais essayer de développer ici les propriétés qui se rapportent aux transformations linéaires des périodes des fonctions $\mathrm{y} x, \mathrm{~g} x, G x, Z x$, dont je me suis occupé dans le Mémoire sur les fonctions doublement périodiques que j'ai donné dans ce Recueil en 1845. Avant d'entrer en matière, je remarque que partant des expressions

$$
\begin{aligned}
& \Omega=\omega+\omega^{\prime} i, \\
& \Upsilon=v+v^{\prime} i,
\end{aligned}
$$

des deux périodes, où $i=\sqrt{-1}$, on obtient, en éerivant

$$
\begin{aligned}
& \Omega^{*}=\omega-\omega^{\prime} i, \\
& \Upsilon^{*}=v-v^{\prime} i,
\end{aligned}
$$

les équations

$$
\begin{aligned}
& \Omega \Upsilon^{*}=\omega v+\omega^{\prime} v^{\prime}-i\left(\omega v^{\prime}-\omega^{\prime} v\right), \\
& \Omega^{*} \Upsilon=\omega v+\omega^{\prime} v^{\prime}+i\left(\omega v^{\prime}-\omega^{\prime} v\right),
\end{aligned}
$$

au moyen desquelles et des valeurs

$$
\beta=\frac{\pi i\left(\omega v^{\prime}-\omega^{\prime} v\right)}{\Omega \Upsilon \bmod .\left(\omega v^{\prime}-\omega^{\prime} v\right)}, \quad B=\frac{\pi\left(\omega v+\omega^{\prime} v^{\prime}\right)}{\Omega \Upsilon \bmod .\left(\omega v^{\prime}-\omega^{\prime} v\right)}
$$

des quantités $\beta, B$, on déduit les formules

$$
B+\beta=\frac{\pi \Omega^{*}}{\Omega \bmod .\left(\omega v^{\prime}-\omega^{\prime} v\right)}, \quad B-\beta=\frac{\pi \Upsilon^{*}}{\Upsilon \bmod .\left(\omega v^{\prime}-\omega^{\prime} v\right)} .
$$


Je ne fais attention qu'aux transformations qui correspondent à des entiers impairs et premiers, et je suppose, de plus, que la transformation soit toujours propre et régulière; c'est-à-dire qu'en écrivant

$$
\begin{aligned}
& (2 k+1) \Omega,=\lambda \Omega+\mu \Upsilon=(\lambda, \mu), \\
& (2 k+1) \Upsilon,=\nu \Omega+\rho \Upsilon=(\nu, \rho),
\end{aligned}
$$

où $2 k+1$ est un entier positif, impair et premier, et où $\lambda, \mu, \nu, \rho$ sont des entiers tels, qu'au signe près, $\lambda \rho-\mu \nu$ soit égal à $2 k+1$, je suppose

$$
\lambda \rho-\mu \nu=2 k+1 \text {, }
$$

(condition pour que la transformation soit propre), et, en outre,

$$
\begin{array}{ll}
\lambda \equiv 1, & \mu \equiv 0,(\bmod .2) \\
\nu \equiv 0, & \rho \equiv 1,
\end{array}
$$

(condition pour que la transformation soit régulière).

On trouve tout de suite

$$
\begin{aligned}
& \Omega=\rho \Omega_{1}-\mu \Upsilon,=(\rho,-\mu)_{1}, \\
& \Upsilon=-\nu \Omega,+\lambda \Upsilon,=(-\nu, \lambda)_{1} ;
\end{aligned}
$$

j'écris aussi

$$
\begin{array}{ll}
\Omega_{1}=\omega_{1}+\omega_{1}^{\prime} i, & \Omega_{1}^{*}=\omega_{1}-\omega_{1}^{\prime} i, \\
\Upsilon=v_{1}+v_{1}^{\prime} i, & \Upsilon_{1}^{*}=v_{1}-v_{1}^{\prime} i,
\end{array}
$$

et je suppose que $B_{\imath}, \beta$, soient des fonctions de $\omega_{\imath}, v$, telles que les fonctions $B, \beta$, de $\omega, v$.

Cela étant, je forme d'abord l'équation

$$
(2 k+1)\left(\omega, v_{\prime}^{\prime}-\omega_{\prime}^{\prime} v_{\imath}\right)=\omega v^{\prime}-\omega^{\prime} v
$$

au moyen de laquelle l'équation

$$
(B+\beta) \Omega=\frac{\pi}{\bmod .\left(\omega v^{\prime}-\omega^{\prime} v\right)} \Omega^{*}
$$

se transforme en

$$
(2 k+1)(B+\beta) \Omega=\frac{\pi}{\bmod .\left(\omega, v_{\prime}^{\prime}-\omega_{\prime}^{\prime} v_{\prime}\right)} \Omega^{*}
$$

De là

$$
\begin{aligned}
& \left\{(2 k+1)(B+\beta)-B_{1}\right\} \Omega=\frac{\pi}{\bmod \left(\omega, v_{1}^{\prime}-\omega_{\prime}^{\prime} v_{1}\right)}\left\{\Omega^{*}-\Omega \frac{\omega_{1} v_{1}+\omega_{\prime}^{\prime} v_{1}^{\prime}}{\Omega, \Upsilon_{1}}\right\}, \\
& =\frac{\pi}{\bmod .\left(\omega_{1} v_{1}^{\prime}-\omega_{\prime}^{\prime} v_{\prime}\right)}\left\{\rho \Omega_{1}^{*}-\mu \Upsilon_{1}^{*}-\frac{\omega_{1} v_{1}+\omega_{\prime}^{\prime} v_{1}^{\prime}}{\Omega, \Upsilon_{1}}\left(\rho \Omega_{1}-\mu \Upsilon_{\imath}\right)\right\},
\end{aligned}
$$

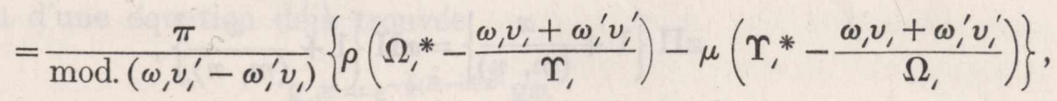

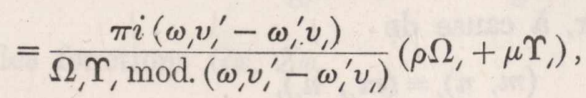


ou enfin

$$
\{\overline{2 k+1}(B+\beta)-B\} \Omega=\quad \beta,(\rho, \mu)
$$

et de même

$$
\{\overline{2 k+1}(B-\beta)-B\} \Omega=-\beta,(\nu, \lambda) ;
$$

équations qui seront bientôt utiles.

Je suppose d'abord que $2 k+1$ soit égal à l'unité, transformation que l'on peut nommer triviale. La fonction $\mathrm{y} x$ est définie par l'équation

$$
\mathrm{y} x=\epsilon^{-\frac{1}{2} B x^{2}} x \Pi\left\{1+\frac{x}{(m, n)}\right\}, \quad \bmod .(m, n)<T, T=\infty ;
$$

dans $(m, n)=m \Omega+n \Upsilon$, les entiers $m, n$ doivent prendre toutes les valeurs positives ou négatives (le seul système $m=0, n=0$ excepté) qui satisfont à l'inégalité

$$
\text { mod. }(m, n)<T \text {, }
$$

dont le second membre $T$ sera ensuite supposé infini. Soit $\mathrm{y}, x$ la fonction correspondante pour les périodes $\Omega, \Upsilon_{\text {, }}$; on aura

$$
\mathrm{y}, x=\epsilon^{-\frac{1}{b} B, x^{2}} x \Pi\left\{1+\frac{x}{(m, n)}\right\}, \quad \bmod .(m, n),<T, \quad T=\infty .
$$

Or

$$
\begin{aligned}
(m, n), & =m \Omega,+n \Upsilon, \\
& =m(\lambda \Omega+\mu \Upsilon)+n(\nu \Omega+\rho \Upsilon), \\
& =(\lambda m+\nu n) \Omega+(\mu m+\rho n) \Upsilon, \\
& =m_{\imath} \Omega+n \Upsilon \Upsilon \\
& =\left(m_{\imath}, n_{\imath}\right) .
\end{aligned}
$$

En écrivant, comme nous venons de le faire,

$$
\begin{aligned}
& m_{l}=\lambda m+\nu n, \\
& n_{\imath}=\nu m+\rho n,
\end{aligned}
$$

on voit tout de suite qu'à chaque système de valeurs entières de $m, n$, correspond un système, et un seul système, de valeurs entières de $m_{t}, n_{i}$; et que de même à chaque système de valeurs entières de $m_{l}, n_{l}$, correspond un système, et un seul système, de valeurs entières de $m, n$; de plus, les systèmes $m=0, n=0$ et $m_{\mathrm{l}}=0, n_{\mathrm{l}}=0$, correspondent l'un à l'autre. Il est donc permis d'écrire

$$
x \Pi\left\{1+\frac{x}{(m, n)}\right\}=x \Pi\left\{1+\frac{x}{(m, n)}\right\},
$$

les limites comme auparavant; car, à cause de

$$
(m, n),=\left(m, n_{l}\right),
$$


la condition pour les limites, savoir:

$$
\text { mod. }(m, n),<T, T=\infty \text {, }
$$

devient

$$
\text { mod. }(m, n)<T, T=\infty \text {. }
$$

Cela donne enfin l'équation

$$
y, x=\epsilon^{-\frac{1}{2}(B,-B) x^{2}} \mathrm{y} x
$$

et, au moyen de cette équation, on obtient une équation correspondante pour la transformation de l'une quelconque des fonctions $\mathrm{y} x, \mathrm{~g} x, G x, Z x$, définies par les équations

$$
\begin{array}{ll}
\mathrm{y} x=\epsilon^{-\frac{1}{3} B x^{2}} \cdot x \Pi\left\{1+\frac{x}{(m, n)}\right\}, & \text { mod. }(m, n)<T, \\
\mathrm{~g} x=\epsilon^{-\frac{1}{-1} B x^{2}} . \quad \Pi\left\{1+\frac{x}{(\bar{m}, n)}\right\}, & \bmod .(\bar{m}, n)<T, \quad T=\infty ; \\
G x=\epsilon^{-\frac{1}{B} B x^{2}} . \Pi\left\{1+\frac{x}{(m, \bar{n})}\right\}, & \bmod .(m, \bar{n})<T, \\
Z x=\epsilon^{-\frac{1}{b} B x^{2}} . \quad \Pi\left\{1+\frac{x}{(\bar{m}, \bar{n})}\right\}, & \bmod (\bar{m}, \bar{n})<T,
\end{array}
$$

(équations dans lesquelles $\bar{m}=m+\frac{1}{2}, \bar{n}=n+\frac{1}{2}$ ). Je prends par exemple la fonction $\mathrm{g} x$, et j'écris dans l'équation entre $\mathrm{y}, x$ et $\mathrm{y} x, x+\frac{1}{2} \Omega$ au lieu de $x$. Soit pour un moment $\rho=2 \rho^{\prime}+1, \mu=2 \mu^{\prime}$; cela donne

$$
x+\frac{1}{2} \Omega=x+\frac{1}{2}(\rho \Omega,-\mu \Upsilon)=x+\left(\bar{\rho}^{\prime},-\mu^{\prime}\right) .
$$

Donc

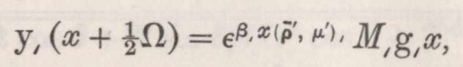

c'est-à-dire

$$
\mathrm{y}_{,}\left(x+\frac{1}{2} \Omega\right)=\epsilon^{\frac{1}{\beta}, x(\rho, \mu)}, M, \mathrm{~g}, x
$$

de plus,

$$
\mathrm{y}\left(x+\frac{1}{2} \Omega\right)=\epsilon^{\frac{1}{\beta} \Omega x} M \mathrm{~g} x .
$$

Ces substitutions étant effectuées, les coefficients $M, M$, doivent être éliminés en écrivant $x=0$; cela donne

$$
\mathrm{g}_{1} x=\epsilon^{-\frac{\dot{s}}{2}(B,-B) x^{2}} \epsilon^{\frac{1}{3} x\left[(B+\beta-B) \Omega-\frac{1}{2} \beta,(\rho, \mu),\right]} \mathrm{g} x,
$$

ou enfin, au moyen d'une équation déjà trouvée,

$$
\mathrm{g}, x=\epsilon^{-\frac{1}{2}\left(B_{i}-B\right) x^{2}} \mathrm{~g} x,
$$

et de même pour les fonctions $G x, Z x$.

C. II. 


\section{4}

DEUXIÈME MÉMOIRE SUR LES FONCTIONS DOUBLEMENT PÉRIODIQUES. on aura

Donc enfin, en représentant par $J x$ l'une quelconque des fonctions y $x, \mathrm{~g} x, G x, Z x$,

$$
J, x=\epsilon^{-\frac{1}{(2}(B,-B) x^{2}} J x
$$

où $J, x$ est ce que devient $J x$ au moyen d'une transformation triviale (propre et régulière) des périodes.

Je passe à présent à la transformation pour un nombre impair et premier $(2 k+1)$ quelconque; mais pour cela on a besoin de connaître la valeur de la fonction

$$
u^{\prime}=\Pi\left\{1+\frac{x}{(m, n)+y}\right\}, \quad \bmod .\{(m, n)+y\}<T, \quad T=\infty,
$$

où $y=a+b i$ est une quantité réelle ou imaginaire quelconque.

Soit $u$ ce que devient $u^{\prime}$ en prenant pour la condition par rapport aux limites

$$
\text { mod. }(m, n)<T, \quad T=\infty \text {; }
$$

on trouve sans peine

$$
u=\epsilon^{\frac{1}{3} B x^{2}+B x y} \frac{\mathrm{y}(x+y)}{\mathrm{y}(y)} .
$$

Pour trouver $u^{\prime}$, je forme l'équation

$$
u: u^{\prime}=\Pi\left\{1+\frac{x}{(m, n)}\right\},
$$

la limite inférieure du produit infini double étant

$$
\text { mod. }\{(m, n)+y\}>T \text {, }
$$

et la limite supérieure

$$
\text { mod. }(m, n)<T, \quad T=\propto ;
$$

cela donne

$$
\begin{aligned}
\log u-\log u^{\prime} & =x \boldsymbol{\Sigma} \frac{1}{(m, n)+y}-\frac{1}{2} x^{2} \boldsymbol{\Sigma} \frac{1}{\{(m, n)+y\}^{2}}+\ldots \\
& =x \boldsymbol{\Sigma} \frac{1}{(m, n)}-\frac{1}{2}\left(x^{2}+2 y x\right) \mathbf{\Sigma} \frac{1}{(m, n)^{2}}+\ldots, \\
& =x \boldsymbol{\Sigma} \frac{1}{(m, n)}
\end{aligned}
$$

car on peut démontrer que

$$
\dot{\mathbf{\Sigma}} \frac{1}{(m, n)^{2}}=0, \quad \boldsymbol{\Sigma} \frac{1}{(m, n)^{3}}=0, \quad \& c .
$$

Pour cela, observons que $m$ et $n$ étant infinis puisque $T$ l'est, la première des sommes dont il s'agit peut se remplacer par l'intégrale double

$$
I=\iint \frac{d m d n}{(m, n)^{2}}
$$


laquelle (en écrivant $m=r \cos \theta, n=r \sin \theta$, ce qui donne, comme on sait, $d m d n=r d r d \theta$ ) devient

$$
I=\iint \frac{d r d \theta}{r(\Omega \cos \theta+r \sin \theta)^{2}}
$$

d'où

$$
I=\iint \frac{(\log r) d \theta}{(\Omega \cos \theta+r \sin \theta)^{2}}
$$

en prenant $(\log r)$ entre les limites convenables. Pour trouver ces limites, j'écris

$$
(m, n)+y=r(\Omega \cos \theta+\Upsilon \sin \theta)+y ;
$$

ce qui donne

$$
\bmod ^{2}\{(m, n)+y\}=\{r(\Omega \cos \theta+\Upsilon \sin \theta)+y\}\left\{r\left(\Omega^{*} \cos \theta+\Upsilon^{*} \sin \theta\right)+y^{*}\right\},
$$

savoir, à l'une des limites

$$
\begin{aligned}
r^{2}(\Omega \cos \theta+\Upsilon \sin \theta) & \left(\Omega^{*} \cos \theta+\Upsilon^{*} \sin \theta\right) \\
+r & \left\{y^{*}(\Omega \cos \theta+\Upsilon \sin \theta)+y\left(\Omega^{*} \cos \theta+\Upsilon^{*} \sin \theta\right)\right\}+T^{2}=0 ;
\end{aligned}
$$

ou, en négligeant les puissances négatives de $T$,

$$
\begin{aligned}
r & =\frac{T}{\sqrt{(\Omega \cos \theta+\Upsilon \sin \theta)\left(\Omega^{*} \cos \theta+\Upsilon^{*} \sin \theta\right)}} \\
& -\frac{1}{2}\left\{\frac{y}{\Omega \cos \theta+\Upsilon \sin \theta}+\frac{y^{*}}{\Omega^{*} \cos \theta+\Upsilon^{*} \sin \theta}\right\},
\end{aligned}
$$

et à l'autre limite,

$$
r=\frac{T}{\sqrt{(\Omega \cos \theta+\Upsilon \sin \theta)\left(\Omega^{*} \cos \theta+\Upsilon^{*} \sin \theta\right)}} .
$$

Or, en représentant ces deux équations par

$$
r=R-\phi, \quad r=R,
$$

on trouve, pour la valeur de $(\log r)$ entre les deux limites,

$$
\log R-\log (R-\phi)=-\log \left(1-\frac{\phi}{R}\right)=0
$$

à cause de la valeur infinie de $R$. Ainsi la somme cherchée est nulle; et il est tout clair que les sommes suivantes $\boldsymbol{\Sigma} \frac{1}{(m, n)^{3}}$, \&c., se réduisent de même à zéro.

Donc enfin,

$$
\log u-\log u^{\prime}=x \sum \frac{1}{(m, n)}
$$


Cela fait voir que

$$
u^{\prime}=\epsilon^{-k x} u,
$$

le coefficient $k$ étant donné au moyen de l'équation

$$
k=\Sigma \frac{1}{(n, m)},
$$

où la somme est prise, comme auparavant, entre les limites

$$
\text { mod. }\{(m, n)+y\}>T, \quad \bmod .(m, n)<T, T=\infty .
$$

Mais il n'est pas permis d'écrire

$$
k=\iint \frac{d m d n}{(m, n)}
$$

En effet, cette intégrale n'est que le premier terme d'une suite dont il faudrait, pour obtenir un résultat exact, prendre deux termes; le second terme de la suite serait une intégrale prise le long d'un contour, et il serait, ce me semble, très-difficile d'en trouver la valeur. Pour: trouver la valeur de $k$, je remarque que $k$ sera fonction linéaire des quantités $T, y, y^{*}, \frac{y^{2}}{T}$, \&c., qui entrent dans les valeurs de $r$; donc, puisqu'en dernière analyse $T=\infty, k$ ne peut être que de la forme $L y+M y^{*}$. Cela étant, en substituant pour $u^{\prime}$ sa valeur, je forme l'équation.

$$
\begin{aligned}
\frac{\mathrm{y}(x+y)}{\mathrm{y}(y)}= & \epsilon^{-\frac{1}{2} B x^{2}} \epsilon^{\left(-B y+L y+M y^{*}\right) x} \cdot \Pi\left\{1+\frac{x}{(m, n)+y}\right\}, \\
& \bmod \{(m, n)+y\}<T, \quad T=\infty,
\end{aligned}
$$

et j'écris successivement

$$
y=\frac{1}{2} \Omega, \quad y=\frac{1}{2} \Upsilon,
$$

ce qui donne pour les valeurs correspondantes du produit infini double $\epsilon^{-\frac{1}{b} B x^{2}}$. gx et $\epsilon^{-\frac{1}{3} B x^{2}} . G x$; en comparant les valeurs ainsi obtenues avec les équations qui donnent les valeurs de $\mathrm{y}\left(x+\frac{1}{2} \Omega\right), \mathrm{y}\left(x+\frac{1}{2} \Upsilon\right)$, on trouve

$$
L=0, \quad M=\frac{\pi}{\bmod .\left(\omega v^{\prime}-\omega^{\prime} v\right)},
$$

ou enfin,

$$
\begin{gathered}
\frac{\mathrm{y}(x+y)}{\mathrm{y}(y)}=\epsilon^{-\frac{1}{3} B x^{2}} \epsilon^{\left(-B y+\frac{\pi}{\bmod .\left(\omega v^{\prime}-\omega^{\prime} v\right)} y^{*}\right) x} . \Pi\left\{1+\frac{x}{(m, n)+y}\right\}, \\
\text { mod. }\{(m, n)+y\}<T, \quad T=\infty,
\end{gathered}
$$

laquelle est l'équation qu'il s'agissait d'établir. Il est à peine nécessaire de faire la remarque que pour $y=0$, on doit considérer à part le facteur $1+\frac{x}{y}$, lequel multiplié par y $(y)$ devient tout simplement $x$; l'équation subsiste donc dans ce cas. 
En revenant au problème des transformations linéaires, partant des équations

$$
\begin{aligned}
& (2 k+1) \Omega,=\lambda \Omega+\mu \Upsilon, \\
& (2 k+1) \Upsilon,=\nu \Omega+\rho \Upsilon,
\end{aligned}
$$

je suppose d'abord que les coefficients $\lambda, \nu$ ne satisfassent pas à la fois aux deux conditions

$$
\lambda \equiv 0, \quad \nu \equiv 0, \quad \bmod .(2 k+1),
$$

et je prends $p, q$ des entiers quelconques tels, que $\lambda p+\nu q$ ne soit pas $\equiv 0, \bmod .(2 k+1)$.

Cela étant, soient

$$
\begin{aligned}
& \lambda p+\nu q=p_{1} \\
& \mu p+\rho q=q_{1} \\
& (2 k+1) \psi=p, \Omega+q, \Upsilon
\end{aligned}
$$

et, par conséquent,

$$
\psi=p \Omega,+q \Upsilon .
$$

Je forme l'équation

$$
\left(m_{l}, n_{l}\right)+s \psi=(m, n)_{l},
$$

savoir

c'est-à-dire

$$
m, \Omega+n_{1} \Upsilon+s \psi=m \Omega_{1}+n \Upsilon_{\prime},
$$

$$
\begin{aligned}
& \lambda m+\nu n-s p_{i}=(2 k+1) m_{\iota}, \\
& \mu m+\nu n-s q_{l}=(2 k+1) n_{\iota},
\end{aligned}
$$

ou, ce qui est la même chose,

$$
\begin{aligned}
m-s p & =m, \rho-n, \nu, \\
n-s q & =m, \mu-n, \lambda .
\end{aligned}
$$

Or, $m, n, s$ étant des entiers donnés, $m, n$ seront aussi des entiers; de même, $m, n$ étant des entiers donnés, on trouve de $k$ à $-k$ un entier $s$ qui donne $m$, un entier. Mais cela étant, $n$, sera aussi un entier; car autrement $n$, serait une fraction ayant pour dénominateur, lequel on voudrait, des nombres $2 k+1, \lambda, \nu$, ce qui est impossible à moins que

$$
\lambda \equiv 0, \quad \nu \equiv 0, \quad \bmod .(2 k+1) .
$$

Mais si ces équations avaient lieu, on trouverait d'abord $s$ de manière à avoir $n$, entier, et alors, puisqu'on n'a pas aussi

$$
\mu \equiv 0, \quad \rho \equiv 0, \quad \bmod .(2 k+1)
$$

(en effet, cela est impossible à cause de l'équation $\lambda \rho-\mu \nu=2 k+1$ ), on démontrerait, comme auparavant, pour $n_{t}$, que $m$, est entier. Donc, enfin, $m, n$ étant des entiers donnés, on trouve pour $m_{t}, n_{t}, s$ un système d'entiers tel que $s$ soit compris de $k$ à $-k$, et l'on voit sans peine qu'il n'y a qu'un seul système de cette espèce. 
A présent, partant de l'équation

$$
\frac{\mathrm{y}(x+y)}{\mathrm{y}(y)}=\epsilon^{-b^{-1 B x^{2}}} \cdot \epsilon^{\left(-B y+\frac{\pi}{\bmod \left(\omega v^{\prime}-\omega^{\prime} v\right)^{y^{2}}}\right) x} \cdot \Pi\left\{1+\frac{x}{\left(m_{\iota}, n_{\iota}\right)+y}\right\}
$$

(et faisant attention à la particularité que présente le cas de $y=0$ ), j'écris successivement

$$
y=0, \quad y= \pm \psi, \ldots, \quad y= \pm k \psi,
$$

et je forme le produit des équations ainsi trouvées. Cela donne, à cause de $\left(m_{\ell}, n_{l}\right)+s \psi$ $=(m, n)$,

$$
\Pi \frac{\mathrm{y}(x+s \psi)}{\mathrm{y}(s \psi)}=\epsilon^{-\frac{1}{(2 k+1)} B x^{2}} \cdot x \Pi\left\{1+\frac{x}{(m, n)_{1}}\right\},
$$

la condition, par rapport aux limites, étant

$$
\text { mod. }(m, n),<T, \quad T=\infty \text {. }
$$

Or

$$
y, x=\epsilon^{-\frac{1}{b} B, x^{2}} \cdot x \Pi\left\{1+\frac{x}{(m, n)}\right\},
$$

avec la même condition, par rapport aux limites; done, enfin,

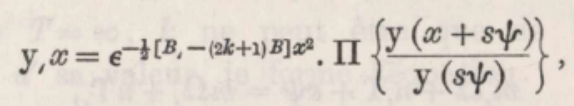

où, dans le numérateur, $s$ doit avoir toutes les valeurs entières depuis $s=-k$ jusqu'à $s=+k$, y compris $s=0$, et dans le dénominateur ces mêmes valeurs, hormis la valeur $s=0$.

Il est, à présent, facile de faire voir que cette propriété subsiste pour l'une quelconque des fonctions $\mathrm{y} x, \mathrm{~g} x, G x, Z x$; en effet, pour la démontrer pour g $x$, j'écris $x+\frac{1}{2} \Omega$ au lieu de $x$; en prenant, pour un moment, $\rho=2 \rho^{\prime}+1, \mu=2 \mu^{\prime}$, cela donne

$$
\begin{gathered}
x+\frac{1}{2} \Omega=+\left(\bar{\rho}^{\prime},=\mu^{\prime}\right), \\
y,\left(x+\frac{1}{2} \Omega\right)=\epsilon^{\beta, x\left(\bar{\rho}^{-}, \mu^{\prime}\right)} \cdot M g, x=\epsilon^{1 \beta, x(\rho, \mu)} \cdot M, g x,
\end{gathered}
$$

c'est-à-dire

$$
\frac{\mathrm{y}_{1}\left(x+\frac{1}{2} \Omega\right)}{\mathrm{y}_{1}\left(\frac{1}{2} \Omega\right)}=\epsilon^{\beta \beta, x(\rho, \mu)}, \mathrm{g}, x .
$$

Or, on déduit de l'expression pour $\mathrm{y}, x$,

$$
\begin{aligned}
& \epsilon^{-1 \beta, x(\rho, \mu)}, \frac{\mathrm{y}_{,}\left(x+\frac{1}{2} \Omega\right)}{\mathrm{y}_{1}\left(\frac{1}{2} \Omega\right)}
\end{aligned}
$$

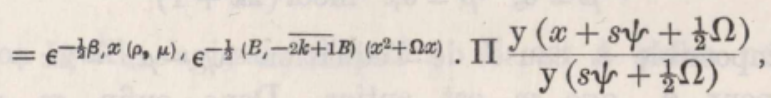

$$
\begin{aligned}
& =\epsilon^{-\frac{1}{1} \beta, x(\rho, \mu)}, \epsilon^{-\frac{1}{1}(B,-\overline{2 k+1} B) x^{2}} \epsilon^{\frac{1}{(2)}(2 k+1) \beta \Omega x} \Pi \frac{\mathrm{g}(x+s \psi)}{\mathrm{g}(s \psi)} ;
\end{aligned}
$$


130] DEUXIÈME MÉMOIRE SUR LES FONCTIONS DOUBLEMENT PÉRIODIQUES. 159

c'est-à-dire

$$
\left.\mathrm{g}, x=\epsilon^{-\frac{1}{2}(B,-\overline{2 k+1} B) x_{2}} \epsilon^{\frac{1}{3} x\{-B, \Omega+2 k+1}(B+\beta) \Omega-\beta,(\varphi, \mu),\right\} \Pi \frac{\mathrm{g}(x+s \psi)}{\mathrm{g}(s \psi)},
$$

ou enfin, à cause de l'équation

$$
-B, \Omega+\overline{2 k+1}(B+\beta) \Omega-\beta,(\rho, \mu),=0,
$$

la valeur de $\mathrm{g}, x$ est

$$
\mathrm{g}, x=\epsilon^{-\frac{1}{2}(B,-2 \overline{k+1} B) x^{2}} \cdot \Pi \frac{\mathrm{g}(x+s \psi)}{\mathrm{g}(s \psi)}:
$$

et en représentant, comme auparavant, l'une quelconque des fonctions $\mathrm{y} x, \mathrm{~g} x, G x, Z x$ par $J x$, on a l'équation

$$
J, x=\epsilon^{-\frac{1}{2}\left(B_{1}-2 \overline{k+1} B\right) x^{2}} \cdot \Pi \frac{J(x+s \psi)}{J(s \psi)},
$$

équation dans laquelle $s$ doit avoir, dans le numérateur, toutes les valeurs entières depuis $s=-k$ jusqu'à $s=k$, y compris $s=0$, et dans le dénominateur, ces mêmes valeurs, hormis la valeur $s=0$.

Je suppose que les valeurs de $p_{t}, q$, soient données (cela va sans dire que l'on ne doit pas avoir à la fois $p, \equiv 0, q, \equiv 0$, mod. $2 k+1)$, et je remarque que l'on a, pour déterminer $\lambda, \mu, \nu, \rho$, les conditions

$$
\begin{aligned}
& \rho p_{1}-\nu q_{1} \equiv 0, \quad \bmod .(2 k+1) \\
&-\mu p_{1}+\lambda q_{1} \equiv 0 \\
& \lambda \equiv 1, \quad \mu \equiv 0, \quad \bmod .2 \\
& \nu \equiv 0, \quad \rho \equiv 1 \\
& \lambda \rho-\mu \nu=2 k+1
\end{aligned}
$$

Et cela étant, on aura ensuite, en rassemblant toutes les équations qui ont rapport à la transformation,

$$
\begin{aligned}
\rho p_{1}-\nu q_{1} & =(2 k+1) p, \\
-\mu p,+\lambda q_{1} & =(2 k+1) q, \\
\Psi & =p, \Omega+q_{1} \Upsilon, \\
(2 k+1) \Omega, & =\lambda \Omega+\mu \Upsilon, \\
(2 k+1) \Upsilon, & =\nu \Omega+\rho \Upsilon .
\end{aligned}
$$


Or, quoique les valeurs de $\lambda, \mu, \nu, \rho$ ne soient pas complétement déterminées au moyen de ces conditions, cependant il est clair que la valeur de la fonction $J, x$ ne dépend que des valeurs de $p_{i}, q$, (en effet, ces valeurs suffisent pour déterminer la quantité $\Psi=p, \Omega+q, \Upsilon$, de laquelle dépend la fonction $J, x)$. Les formes différentes de $J, x$, pour les systèmes de valeurs de $\lambda, \mu, \nu, \rho$, qui correspondent à des valeurs données de $p_{l}, q_{l}$, doivent donc se dériver de l'une quelconque de ces formes, au moyen d'une transformation triviale des modules $\Omega_{1}, \Upsilon$, Il est, de plus, clair que les valeurs de $p_{1}, q_{1}$, qui sont égales à des multiples de $(2 k+1)$ près, ne donnent qu'une seule valeur de $J, x$. Je suppose d'abord que

$$
p_{1} \equiv 0, \quad \bmod .(2 k+1),
$$

on peut trouver un entier $\theta$ tel que

$$
\theta p, \equiv 1, \quad \bmod .(2 k+1)
$$

en prenant alors

$$
\theta q, \equiv q, \quad \text { mod. }(2 k+1)
$$

cela donne

$$
\theta(p, \Omega+q, \Upsilon) \equiv \Omega+q, \Upsilon, \quad \bmod .(2 k+1)
$$

savoir

$$
\theta \Upsilon \equiv \Omega+q \Upsilon \Upsilon \bmod .(2 k+1)
$$

Mais en donnant à $s$ des valeurs entières quelconques, depuis $-k$ jusqu'à $k$, le système des valeurs de $s \psi$ est équivalent au système des valeurs de $s \theta \psi$, mod. $(2 k+1)$; il est donc permis d'écrire, sans perte de généralité,

$$
\Psi=\Omega+q \Upsilon \Upsilon
$$

De même pour

$$
p_{1} \equiv 0, \quad \bmod (2 k+1)
$$

on démontre que l'on peut donner à $q$, une valeur quelconque, sans changer pour cela la valeur de $J, x$; il convient d'avoir $p$, impair et $q$, pair. J'écris donc, pour le premier cas, $2 q$, au lieu de $q$, et je suppose que, dans le deuxième cas, les valeurs de $p_{\imath}, q$, soient

$$
p_{1}=2 k+1, \quad q,=2
$$

Cela donne:

\section{Premier cas.}

$$
\Psi=\Omega+2 q, \Upsilon,
$$

$\mathrm{q}$, un entier quelconque, $\mathrm{y}$ compris zéro, depuis $-k$ jusqu'à $+k$.

Deuxième cas.

$$
\Psi=(2 k+1) \Omega+2 \Upsilon ;
$$

le nombre des valeurs différentes de $\Psi$ sera donc, en tout, $2 k+2$. 
On obtient tout de suite, pour le premier cas, le système d'équations

$$
\begin{gathered}
p_{\imath}=1, \quad q=2 q, \\
\lambda=1, \quad \mu=2 q, \\
\nu=0, \quad \rho=(2 k+1), \\
p=1, \quad q=0 \\
\left\{\begin{array}{l}
\psi=\frac{1}{2 k+1}(\Omega+2 q, \Upsilon), \\
\Omega=\frac{1}{2 k+1}(\Omega+2 q, \Upsilon)=\psi, \\
\Upsilon,=\Upsilon .
\end{array}\right.
\end{gathered}
$$

Le cas particulier le plus simple est celui de $q_{,}=0$; cela donne

et, de là,

$$
\psi=\Omega_{i}=\frac{1}{2 k+1} \Omega, \quad \Upsilon,=\Upsilon
$$

$$
\frac{\Omega_{1}}{\Upsilon_{1}}=\frac{1}{2 k+1} \frac{\Omega}{\Upsilon}
$$

et même le cas général se réduit à celui-ci, car, au moyen d'une transformation triviale, on obtiendrait

et puis

$$
\Omega^{\prime}=\Omega+2 q, \Upsilon, \quad \Upsilon^{\prime}=\Upsilon,
$$

$$
\psi=\Omega,=\frac{1}{2 k+1} \Omega^{\prime}, \quad \Upsilon,=\Upsilon^{\prime},
$$

et, de là,

$$
\frac{\Omega_{\prime}}{\Upsilon_{1}}=\frac{1}{2 k+1} \frac{\Omega^{\prime}}{\Upsilon^{\prime}} .
$$

Les équations correspondantes pour le deuxième cas sont:

$$
\begin{aligned}
& p=2 k+1, \quad q=2, \\
& \lambda=2 k+1, \quad \mu=0, \\
& \nu=0, \quad \rho=1, \\
& p=1, \quad q=2, \\
& \psi=\frac{1}{2 k+1}\{(2 k+1) \Omega+2 \Upsilon\} \\
& \Omega_{1}=\Omega, \\
& \Upsilon=\frac{1}{2 k+1} \Upsilon
\end{aligned}
$$

ce qui donne

$$
\frac{\Omega_{\prime}}{\Upsilon_{1}}=(2 k+1) \frac{\Omega}{\Upsilon} .
$$

c. II. 
J'ajoute, sans m'arrêter pour les démontrer, quelques formules de transformation pour le nombre 2; je trouve d'abord

$$
\begin{aligned}
& \Omega_{l}=\frac{1}{2} \Omega_{l}, \quad \Upsilon=\Upsilon, \\
& \left\{\begin{array}{l}
\mathrm{y}_{f} x=\epsilon^{-\frac{1}{2}\left(B_{,}-2 B\right) x^{2}} \mathrm{y} x \mathrm{~g} x, \\
\mathrm{~g}_{f} x=\epsilon^{-\frac{1}{2}\left(B_{,}-2 B\right) x^{2}} \frac{\mathrm{g}\left(x-\frac{1}{4} \Omega\right) \mathrm{g}\left(x+\frac{1}{4} \Omega\right)}{\mathrm{g}^{2}\left(\frac{1}{4} \Omega\right)}, \\
G_{,} x=\epsilon^{-\frac{1}{2}\left(B_{,}-2 B\right) x^{2}} G x Z x, \\
Z_{,} x=\epsilon^{-\frac{1}{2}\left(B_{i}-2 B\right) x^{2}} \frac{Z\left(x-\frac{1}{4} \Omega\right) Z\left(x+\frac{1}{4} \Omega\right)}{Z^{2}\left(\frac{1}{4} \Omega\right)}
\end{array}\right.
\end{aligned}
$$

Ces équations donnent, en introduisant les fonctions elliptiques, $\phi x, f x, F x$ données au moyen de

$$
\phi x=\frac{\mathrm{y} x}{Z x}, \quad f x=\frac{g x}{Z x}, \quad F x=\frac{G x}{Z x},
$$

les équations

$$
\begin{gathered}
\frac{\phi, x}{F, x}=\phi x \frac{f x}{F x}, \\
f, x=\frac{f\left(x-\frac{1}{4} \Omega\right) f\left(x+\frac{1}{4} \Omega\right)}{f^{2}\left(\frac{1}{4} \Omega\right)},
\end{gathered}
$$

dont la seconde peut encore s'écrire sous la forme

$$
f, x=\frac{1-c^{2} \frac{F^{2}\left(\frac{1}{4} \Omega\right)}{f^{2}\left(\frac{1}{4} \Omega\right)} \phi^{2} x}{1+e^{2} c^{2} \phi^{2}\left(\frac{1}{4} \Omega\right) \phi^{2} x},
$$

et les deux équations combinées ensemble conduisent sans peine à la valeur des modules $c_{l}, e_{l}$. On trouve en effet, en mettant comme à l'ordinaire $b^{2}=c^{2}+e^{2}$,

et puis

$$
\begin{aligned}
& c_{1}^{2}=4 b c, \\
& e_{,}^{2}=(b-c)^{2},
\end{aligned}
$$

$$
\begin{aligned}
\phi, x & =\frac{1-c(c-b) \phi^{2} x}{\phi x f x}, \\
f, x & =\frac{1-c(c+b) \phi^{2} x}{1-c(c-b) \phi^{2} x}, \\
F, x & =\frac{F x}{1-c(c-b) \phi^{2} x},
\end{aligned}
$$

formules qui correspondent à celles de la transformation de Lagrange. Les équations pour $y, x, Z, x$ donnent encore une valeur de $\phi, x$, laquelle, égalée à la valeur qui vient d'être trouvée, donne

$$
\frac{\mathrm{y} x \mathrm{~g} x Z^{2}\left(\frac{1}{4} \Omega\right)}{Z\left(x-\frac{1}{4} \Omega\right) Z\left(x+\frac{1}{4} \Omega\right)}=\frac{\phi x f x}{1-c(c-b) \phi^{2} x} .
$$


130] DEUXIÈME MÉMOIRE SUR LES FONCTIONS DOUBLEMENT PÉRIODIQUES. 163

On obtient tout de suite les formules pour la transformation analogue $\Omega_{1}=\Omega, \Upsilon_{,}=\frac{1}{2} \Upsilon$. Mais il faut de plus considérer le système

$$
\Omega,=\frac{1}{2}(\Omega-\Upsilon), \quad \Upsilon=\frac{1}{2}(\Omega+\Upsilon):
$$

on aura alors

et puis, en écrivant

$$
\left\{\begin{aligned}
\mathrm{y}, x & =\epsilon^{-\frac{1}{2}\left(B_{,}-2 B\right) x^{2}} \mathrm{~g} x Z x, \\
\mathrm{~g}, x & =\epsilon^{-\frac{1}{2}\left(B_{-}-2 B\right) x^{2}} \frac{\mathrm{y}\left(x+\frac{1}{4} \overline{\Omega-\Upsilon}\right) \mathrm{y}\left(x-\frac{1}{4} \overline{\Omega-\Upsilon}\right)}{-\mathrm{y}^{2}\left(\frac{1}{4} \overline{\Omega-\Upsilon}\right)} \\
& =\epsilon^{-\frac{1}{2}\left(B_{-}-2 B\right) x^{2}} \frac{Z\left(x+\frac{1}{4} \overline{\Omega-\Upsilon}\right) Z\left(x+\frac{1}{4} \overline{\Omega-\Upsilon}\right)}{Z^{2}\left(\frac{1}{4} \overline{\Omega-\Upsilon}\right)}, \\
G, x & =\epsilon^{-\frac{1}{1}\left(B_{-}-2 B\right) x^{2}} \frac{\mathrm{y}\left(x+\frac{1}{4} \overline{\Omega+\Upsilon}\right) \mathrm{y}\left(x-\frac{1}{4} \overline{\Omega+\Upsilon}\right)}{-\mathrm{y}^{2}\left(\frac{1}{4} \overline{\Omega+\Upsilon}\right)} \\
& =\epsilon^{-\frac{1}{2}(B-2 B) x^{2}} \frac{Z\left(x+\frac{1}{4} \overline{\Omega+\Upsilon}\right) Z\left(x-\frac{1}{4} \overline{\Omega+\Upsilon}\right)}{Z^{2}\left(\frac{1}{4} \overline{\Omega+\Upsilon}\right)} \\
Z, x & =\epsilon^{-\frac{1}{2}\left(B_{1}-2 B\right) x^{2}} \mathrm{~g} x G x ;
\end{aligned}\right.
$$

on obtient

$$
c_{,}{ }^{2}=(e-i c)^{2}, \quad-e_{,}^{2}=(e+i c)^{2}
$$

$$
\begin{aligned}
\phi, x & =\frac{\phi x}{f x F x}, \\
f, x & =\frac{1+i c e \phi^{2} x}{f x F^{\prime} x}, \\
F, x & =\frac{1-i c e \phi^{2} x}{f x F^{\prime} x} ; \\
\frac{1+i c e \phi^{2} x}{f x F x} & =\frac{Z\left(x+\frac{1}{4} \overline{\Omega-\Upsilon}\right) Z\left(x-\frac{1}{4} \overline{\Omega-\Upsilon)}\right.}{Z^{2}\left(\frac{1}{4} \overline{\Omega-\Upsilon}\right) \mathrm{g} x G x}, \\
\frac{1-i c e \phi^{2} x}{f x F^{\prime} x}=\frac{Z\left(x+\frac{1}{4} \overline{\Omega+\Upsilon}\right) Z\left(x-\frac{1}{4} \overline{\Omega+\Upsilon}\right)}{Z^{2}\left(\frac{1}{4} \overline{\Omega+\Upsilon}\right) \mathrm{g} x G x} & \\
\frac{1+i c e \phi^{2} x}{1-i c e \phi^{2} x}= & \frac{Z\left(x+\frac{1}{4} \overline{\Omega-\Upsilon}\right) Z\left(x-\frac{1}{4} \overline{\Omega-\Upsilon}\right) Z^{2}\left(\frac{1}{4} \overline{\Omega+\Upsilon}\right)}{Z\left(x+\frac{1}{4} \overline{\Omega+\Upsilon}\right) Z\left(x-\frac{1}{4} \overline{\Omega-\Upsilon}\right) Z^{2}\left(\frac{1}{4} \overline{\Omega-\Upsilon}\right)}
\end{aligned}
$$

ou, au moins, ces formules seront exactes au signe de $i$ près; car il serait peut-être difficile de déterminer quel est le signe qu'on doit donner à cette quantité. 